University of Nebraska - Lincoln

DigitalCommons@University of Nebraska - Lincoln

Faculty Publications, Department of Psychology

Psychology, Department of

December 2001

\title{
Multimodal assessment of the effect of chewing gum on nicotine withdrawal
}

Lee M. Cohen

Texas Tech University, Lubbock, TX, USA

Dana M. Britt

Oklahoma State University

Frank L. Collins

Oklahoma State University

Mustafa al-Absi

University of Minnesota School of Medicine

Dennis E. McChargue

University of Nebraska-Lincoln, dmcchargue2@unl.edu

Follow this and additional works at: https://digitalcommons.unl.edu/psychfacpub

Part of the Psychiatry and Psychology Commons

Cohen, Lee M.; Britt, Dana M.; Collins, Frank L.; al-Absi, Mustafa; and McChargue, Dennis E., "Multimodal assessment of the effect of chewing gum on nicotine withdrawal" (2001). Faculty Publications, Department of Psychology. 257.

https://digitalcommons.unl.edu/psychfacpub/257

This Article is brought to you for free and open access by the Psychology, Department of at DigitalCommons@University of Nebraska - Lincoln. It has been accepted for inclusion in Faculty Publications, Department of Psychology by an authorized administrator of DigitalCommons@University of Nebraska - Lincoln. 


\title{
Multimodal assessment of the effect of chewing gum on nicotine withdrawal
}

\author{
Lee M. Cohen ${ }^{\mathrm{a}, \mathrm{b}, *}$, Dana M. Britt ${ }^{\mathrm{b}, \mathrm{c}}$, Frank L. Collins ${ }^{\mathrm{b}}$, Mustafa al'Absi ${ }^{\mathrm{d}}$, \\ Dennis E. McChargue ${ }^{\mathrm{e}}$ \\ ${ }^{a}$ Department of Psychology, Texas Tech University, Lubbock, TX, USA \\ ${ }^{\mathrm{b}}$ Department of Psychology, Oklahoma State University, Stillwater, OK, USA \\ ${ }^{\mathrm{c}}$ Center for Behavioral and Preventive Medicine, Brown University, Providence, RI USA \\ ${ }^{\mathrm{d}}$ Department of Behavioral Sciences, University of Minnesota School of Medicine, Duluth, MN, USA \\ ${ }^{\mathrm{e}}$ University of Illinois at Chicago and the Hines VA Hospital, Chicago, IL, USA
}

\begin{abstract}
The present study was designed to evaluate the usefulness of chewing gum to reduce nicotine withdrawal, craving, and salivary cortisol concentrations during temporary nicotine deprivation. A total of 20 male smokers were studied under conditions when gum was and was not accessible during a 4-hour deprivation period. All subjects smoked an initial cigarette shortly after arrival for the two experimental sessions and were informed that they would be unable to smoke for the remainder of each session. The sessions consisted of each subject watching a movie, then waiting in the lab for two consecutive 30-min intervals. Self-reported nicotine withdrawal and craving were assessed four times and salivary cortisol five times during each experimental session. Results from this study indicate that chewing gum helps with self-reported withdrawal but not craving when a smoker is prevented from smoking. This study also provides preliminary data on the use of salivary cortisol as a physiological marker that may map these self-reports of nicotine withdrawal and craving. (C) 2001 Elsevier Science Ltd. All rights reserved.
\end{abstract}

Keywords: Nicotine; Withdrawal; Gum; Cortisol

\section{Introduction}

It is well documented that cigarette smoking is associated with a variety of serious cardiovascular and pulmonary problems, making smoking the number one most preventable

* Corresponding author. Texas Tech University, Department of Psychology, Box 42051, Lubbock, TX 494092051, USA. 
cause of death in America (U.S. Department of Health and Human Services, 1990). Despite the well-known health hazards, more than 50 million Americans continue to use tobacco products (U.S. Department of Health and Human Services, 1988). Thus, smoking cessation could prevent a large number of deaths each year and defer the onset of a large number of terminal illnesses. Many smokers find it difficult to stop using cigarettes, however, and this is confirmed by the staggering rate that ex-smokers relapse. Of the 17 million smokers that try to quit each year, fewer than 1 out of 10 actually succeed (Kessler, 1994).

It is widely acknowledged that a cluster of symptoms is observed following smoking cessation, and these symptoms are largely due to the effects of nicotine withdrawal (U.S. Department of Health and Human Services, 1988), which may be one of the main reasons that smokers relapse (Gross \& Stitzer, 1989). Elevated levels of stress associated with craving and nicotine withdrawal symptoms may also increase the likelihood of relapse (Stitzer \& Gross, 1988). Although many smoking cessation protocols incorporate alternative behavioral activities during abstinence, empirical evidence has not reliably shown that these behavioral components influence nicotine withdrawal or craving symptoms (Hajek, 1994; Hughes, 1993).

Recent studies conducted in our laboratory have demonstrated that simple alternatives to smoking, such as chewing gum, may influence urge to smoke and nicotine withdrawal (Cohen, Britt, Collins, Stott, \& Carter, 1999; Cohen, Collins, \& Britt, 1997). Cohen et al. (1997) assessed the effects of temporary deprivation (approximately 3 hours) on nicotine withdrawal and craving symptoms. When smokers were instructed to use chewing gum, their self-reported nicotine withdrawal and craving symptoms substantially declined compared to a situation where they did not have access to gum. However, one criticism of the aforementioned study is that the data collected were entirely subjective (i.e., self-report). The validation of a physiological marker that maps these self-reported changes would help further substantiate these findings.

The present study was designed to evaluate the usefulness of chewing gum to reduce nicotine withdrawal, craving, and salivary cortisol concentrations during short-term nicotine deprivation. It was predicted that nicotine deprivation would elicit increases over time in nicotine withdrawal, craving, and salivary cortisol concentrations. Moreover, it was hypothesized that when smokers were asked to chew gum during a period of brief nicotine deprivation, they should show significantly lower salivary cortisol levels and report fewer nicotine withdrawal and craving symptoms compared to a period of nicotine deprivation when they did not have access to gum.

\section{Method}

\subsection{Subjects}

A total of 20 Caucasian male undergraduate smokers participated in this study. Participants were approximately 23 years of age $(M=22.95$, S.D. $=6.73$, range $18-42)$, reported smoking just under 21 cigarettes per day $(M=20.95$, S.D. $=4.10$, range 16-30), and averaged almost a score of 6 on the Fagerström (Fagerström, 1978; $M=5.94$, S.D. =1.56, range 4-9). 


\subsection{Materials}

\subsubsection{Fagerström tolerance questionnaire (FTQ; Fagerström, 1978)}

The FTQ is an eight-item self-report measure that is designed to assess various components of smoking behavior, including an estimate of daily intake, difficulty in refraining, and other aspects related to the pattern of intake. The FTQ has a scoring range of $0-11$ points, with a score of 0 assumed to be indicative of minimum nicotine dependence and a score of 11 indicative of maximum nicotine dependence.

\subsubsection{General habit information (GHI)}

The GHI is a self-report questionnaire designed specifically for this study to obtain information regarding personal habits that might influence a subject's cortisol levels. This questionnaire examines sleep habits, smoking habits, caffeine consumption, and medical information.

\subsubsection{Withdrawal symptom checklist (WSC; Hughes \& Hatsukami, 1986)}

The WSC is a 12-item self-report measure that is designed to assess the presence of tobacco withdrawal symptoms and the severity of each symptom. The severity scores are based on a four-point Likert scale, ranging from 0 (not present) to 3 (severe). The instrument has been shown to be valid and reliable.

\subsection{Procedure}

Subjects attended a brief adaptation session (approximately $25 \mathrm{~min}$ ) and two experimental sessions. At the beginning of each experimental session, subjects relaxed for approximately $20 \mathrm{~min}$. They were then asked to provide a small sample of their saliva and to smoke a cigarette. Upon completion of the cigarette, subjects were again asked to relax for approximately $20 \mathrm{~min}$ and were asked to rate their current withdrawal symptoms by completing the WSC (Baseline). For the remainder of the session, subjects had no access to cigarettes until the completion of the protocol. Subjects provided a second saliva sample and selected a movie from a list. Upon completion of the movie, subjects again completed the WSC (Time 1) and provided a third saliva sample. Subjects were then asked to remain in the lab and read magazines of a neutral subject matter (i.e., Newsweek, Times, and Sports Illustrated) for 1 hour. Half-way through the hour period, subjects were asked to fill out the WSC (Time 2) and provided a fourth sample of saliva. At the end of the hour period, subjects were again asked to fill out the WSC (Time 3) and provided a fifth and final sample of saliva.

Subjects participated in this protocol on two occasions separated by 2-14 days. On one occasion, subjects were instructed to chew at least one piece of non-nicotine, sugarless chewing gum at the start of the movie and were informed that they could chew as much chewing gum as they wished until the termination of the protocol. A variety of flavors of the same brand of sugarless gum was left in the room with each subject. On the other occasion, participants did not have access to chewing gum at any point during the session. The Gum/ No-Gum sessions were counterbalanced across the two groups. Movies chosen for both 
experimental sessions were approximately the same length (within $15 \mathrm{~min}$ of each other) and were classified as the same type of movie (e.g., drama, action/adventure). The magazines available during both of the experimental sessions were the same.

\subsubsection{Saliva/cortisol collection}

Salivary cortisol was collected on five occasions during each of the experimental sessions. Saliva samples were collected using a commercially available collection device (Salivette ${ }^{\mathbb{R}}$, Sarstedt; Rommelsdorf, Germany) and were centrifuged, then stored at $-70^{\circ} \mathrm{C}$ until assayed.

\subsection{Experimental design}

All analyses used a within-subject design where each subject served as his own control. Analyses were conducted for each of the dependent variables with the family-wise $\alpha$ level set at $p<.05$.

\section{Results}

\subsection{Craving effects}

Three planned contrasts were conducted to test whether gum had the hypothesized ameliorative effect on craving at Times 1,2 , and 3 . In order to maintain the experiment-

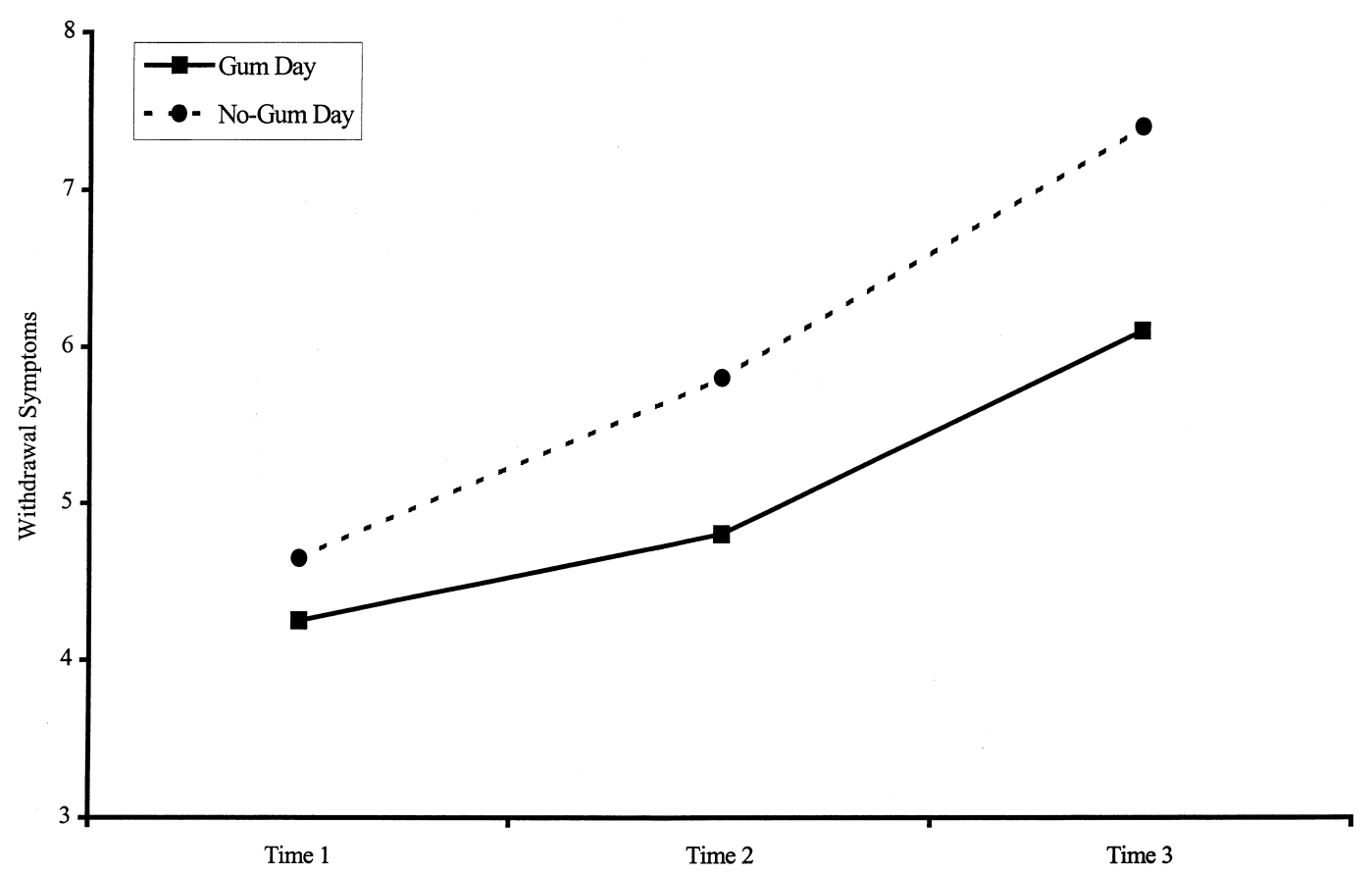

Fig. 1. Mean total withdrawal score for Gum and No-Gum conditions at Times 1-3. 
wise Type I error rate at $\alpha=0.05$, a Bonferroni correction for three tests was utilized, resulting in an error rate per contrast of $p=.016$. None of the hypothesized contrasts were significant.

\subsection{Nicotine withdrawal effects}

Planned contrasts were again carried out (Bonferroni-adjusted $p=.016$ ) to test whether gum had the hypothesized ameliorative effect on nicotine withdrawal at Times 1, 2, and 3. At Time 1, no significant difference was observed between the Gum and No-Gum conditions $[F(1,57)=0.86$, n.s.]. However, as the nicotine deprivation period progressed, significant differences were observed. At Time 2, the Gum condition was associated with lower reported levels of withdrawal when compared to the No-Gum condition $[F(1,57)=5.39, p<.01]$. At Time 3, the effect was even more pronounced, with the Gum condition again demonstrating significantly fewer withdrawal symptoms when compared to the No-Gum condition $[F(1,57)=9.11, p<.01]$. These results are depicted in Fig. 1 .

\subsection{Cortisol effects}

Cortisol levels did not change between days as a function of the use of gum, suggesting that the use of chewing gum did not alter cortisol levels in this sample $[F(2,38)=1.15$, n.s.].

\section{Discussion}

The results of this study are consistent with a previous study, which showed that chewing gum helps with nicotine withdrawal when a nicotine-dependent person was prohibited from smoking (Cohen et al., 1997). When smokers were asked to chew gum, they reported significantly less withdrawal as compared to sessions where they were not permitted to chew gum. In the current study, as the withdrawal period lengthened, differences between the two experimental sessions (Gum Day and No-Gum Day) became more pronounced. Caution should be used, however, when interpreting the meaning of this statement. The results do not suggest that chewing gum will allow a person to avoid withdrawal altogether. Withdrawal was clearly reported by the subjects who participated in this study, yet, it was observed less acutely in subjects when they were instructed to chew gum.

Surprisingly, when subjects were instructed to chew gum in the present study, they did not report less craving for a cigarette compared to the sessions in which they were not permitted to chew gum. This finding is inconsistent with previous research showing reduced craving symptoms when subjects chewed gum during temporary nicotine deprivation (Cohen et al., 1997) but is consistent with other nicotine research showing no significant changes in craving when a behavioral alternative was accessible (McChargue, Collins, \& Cohen, 1996). These apparent inconsistencies may reflect the multidimensional nature of craving, which is influenced by appetitive, conditioned tolerance, affect, and withdrawal components of nicotine dependence (Rohsenow, Niaura, Childress, Abrams, \& Monti, 1991). Essentially, behavioral alternatives during brief nicotine deprivation may not be adequate to influence the 
experience of craving for nicotine because craving may be triggered by factors other than withdrawal, such as appetitive processes.

Finally, this study provides preliminary data on the use of salivary cortisol as a potential physiological marker for tracking nicotine withdrawal. However, changes in cortisol levels are slow to observe, thus, perhaps, a study that extends the period of withdrawal would show stronger findings.

Despite the encouraging findings, results should be interpreted with caution. Results from the current study were based on young, white, college students who were not actively trying to quit smoking. Thus, the extent to which these findings can be generalized to heavier smokers, older smokers, smokers of varying ethnicities, or even female smokers has yet to be examined. Second, the present study does not examine the long-term effectiveness of chewing gum as an aid for reducing the withdrawal symptoms in a population of smokers. Conclusions drawn from this study are limited to individuals experiencing acute withdrawal only.

These results suggest that a behavioral alternative, such as gum, can produce a significant reduction in one's total withdrawal but does little to influence craving for nicotine. This study, coupled with previous findings, suggests that clinicians should encourage the use of behavioral alternatives during periods of brief abstinence.

\section{Acknowledgments}

The current study is based on the dissertation of the first author and was conducted at Oklahoma State University. Appreciation is extended to Mark G. Myers, Ph.D. and John F. Kelly for their reviews of an earlier version of this manuscript. Special appreciation is extended to William R. Lovallo, Ph.D. and Barbara S. McKey, RN of the University of Oklahoma Health Sciences Center, Behavioral Sciences Lab who assisted with the cortisol sample preparation and storage. The saliva cortisol assays were performed by M. L'HermiteBaleriaux, Universite Libre de Bruxelles, Belgium.

\section{References}

Cohen, L. M., Britt, D. M., Collins, F. L. Jr., Stott, H., \& Carter, L. (1999). Chewing gum affects smoking topography. Experimental and Clinical Psychopharmacology, 7, 444-447.

Cohen, L. M., Collins, F. L., \& Britt, D. M. (1997). The effect of chewing gum on tobacco withdrawal. Addictive Behaviors, 22, 769-773.

Fagerström, K. O. (1978). Measuring degree of physical dependence to tobacco smoking with reference to individualization of treatment. Addictive Behaviors, 3, 235-241.

Gross, J., \& Stitzer, M. L. (1989). Nicotine replacement: ten-week effects on tobacco withdrawal symptoms. Psychopharmacology, 98, 334-341.

Hajek, P. (1994). Treatments for smokers. Addiction, 89, 1543-1549.

Hughes, J. R. (1993). Pharmacotherapy for smoking cessation: unvalidated assumptions, anomalies, and suggestions for future research. Journal of Consulting and Clinical Psychology, 61, 751-760.

Hughes, J. R., \& Hatsukami, D. K. (1986). Signs and symptoms of tobacco withdrawal. Archives of General Psychiatry, 43, 289-294. 
Kessler, D. A. (Commissioner of Food and Drugs) (1994). Statement on nicotine-containing cigarettes. Statement delivered to the House Subcommittee on Health and the Environment (March 25).

McChargue, D. E., Collins, F. L., \& Cohen, L. M. (1996). Differentiating withdrawal patterns related to smokeless tobacco users and smokers. Poster presented at the Association for the Advancement of Behavior Therapy Conference, New York, NY (November).

Rohsenow, D. J., Niaura, R. S., Childress, A. R., Abrams, D. B., \& Monti, P. M. (1991). Cue reactivity in addictive behaviors: theoretical and treatment implications. The International Journal of Addictions, 25, 957-993.

Stitzer, M. L., \& Gross, J. (1988). Smoking relapse: the role of pharmacological and behavioral factors. In: O. F. Pomerleau, \& C. S. Pomerleau (Eds.), Nicotine replacement: a clinical evaluation (pp. 163-184). New York: Liss.

US Department of Health and Human Services. (1988). The health consequences of smoking: nicotine addiction. Washington DC: Government Printing Office (DHHS Publication No. CDC 88-8406).

US Department of Health and Human Services. (1990). Smoking and health: a national status report. Washington, DC: Government Printing Office (DHHS Publication No. CDC 87-8396). 